\title{
Mikrostrukturielle Charakterisierung von ultra-hochfesten Schweißgütern
}

\author{
Phillip Haslberger ${ }^{1}$, Wolfgang Ernst ${ }^{2}$ und Ronald Schnitzer ${ }^{1,3}$ \\ ${ }^{1}$ Department Metallkunde und Werkstoffprüfung, Montanuniversität Leoben, Leoben, Österreich \\ ${ }^{2}$ voestalpine Stahl Linz GmbH, Linz, Österreich \\ ${ }^{3}$ voestalpine Böhler Welding Austria $\mathrm{GmbH}$, Kapfenberg, Österreich
}

Eingegangen 15. April 2016; angenommen 25. April 2016; online publiziert 20. Juni 2016

\begin{abstract}
Zusammenfassung: Hochfeste Schweißkonstruktionen sind entscheidend für die Realisierung von Leichtbaukonstruktionen für verschiedenste Industriesegmente. Unterschiedliche Methoden wurden verwendet, um die Mikrostruktur von ultra-hochfesten Schweißgütern mit unterschiedlicher chemischer Zusammensetzung zu charakterisieren. Dadurch sollte es möglich sein, die Korngröße mit der Zähigkeit zu korrelieren.
\end{abstract}

Schlüsselwörter: Schweißgut, Mikrostruktur, Korngröße, Lichtmikroskopie, EBSD

Microstructural Characterization of Ultra-high Strength All-weld Metal

Abstract: Welded steel structures are a crucial part of lightweight constructions in several industrial segments. The filler metal plays a vital role in optimizing the properties of welded joints. In this study different methods were used to characterize the microstructure of ultra-high strength allweld metal samples with different chemical compositions. It was evaluated which method can be used to link the grain size of a sample to its toughness.

Keywords: Weld metal, Microstructure, Grain size, Light optical microscopy, EBSD

\section{Einleitung}

In der Stahl verarbeitenden Industrie besteht ein ständiger Bedarf an Schweißkonstruktionen mit reduziertem Ge-

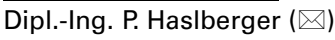

Department Metallkunde und Werkstoffprüfung,

Montanuniversität Leoben,

Roseggerstraße 12/2,

8700 Leoben, Österreich

E-Mail: phillip.haslberger@unileoben.ac.at
}

wicht bei zumindest gleichbleibender Tragfähigkeit. Je nach Schweißverfahren spielt auch der Schweißzusatz eine entscheidende Rolle bei der Bestimmung der mechanischen Eigenschaften des geschweißten Bauteils. Eine nachträgliche Wärmebehandlung des Schweißguts ist dabei oft aus Kostengründen unerwünscht. Besonders das auf den Grundwerkstoff abgestimmte Legierungskonzept des Schweißzusatzes soll deswegen dazu beitragen, ein optimales Verhältnis von Festigkeit und Zähigkeit einzustellen [1].

Für hochfeste, thermomechanisch behandelte oder vergütete Bleche wird zurzeit ein martensitisch erstarrender Schweißzusatz mit einer Festigkeit > $1100 \mathrm{MPa}$ entwickelt. Eine tiefgehende mikrostrukturielle Charakterisierung des Schweißguts ist dabei unerlässlich, um die Beziehung zwischen Mikrostruktur und Eigenschaften zu verstehen. Neben der klassischen Lichtmikroskopie sind hier auch Methoden mit höherer Auflösung wie Raster-Elektronenmikroskopie (REM) oder Elektronen-Rückstreubeugung (= "Electron Backscatter Diffraction“, kurz: EBSD) hilfreich.

Vor allem die Anwendung von EBSD vertiefte das Verständnis des mikrostrukturiellen Aufbaus von Martensit [2-7]. Es wurde festgestellt, dass ein Austenitkorn in sog. "packets" unterteilt werden kann, das wiederum in "blocks“ unterteilt wird. Diese "blocks“ enthalten mehrere einzelne Martensitlatten mit geringer kristallographischer Missorientierung.

Viele martensitische Stähle wurden in dieser Hinsicht ausführlich untersucht, es gibt aber wenig Literatur zur Charakterisierung von martensitischem Schweißgut. W. Vanovsek lieferte hier Ansätze, die primäre dendritische Struktur mithilfe einer Pikrinsäure-Ätzung sichtbar zu machen und die ehemaligen Austenitkörner nach einer Nital-Ätzung auszuwerten $[8,9]$.

Im Rahmen dieser Arbeit soll weiterführend untersucht werden, welche Methoden eine optimale Charakterisierung der vorliegenden Mikrostruktur in martensitischem Schweißgut ermöglichen. 


\section{Experimentelle Durchführung}

Für die Versuche wurden Proben aus reinem Schweißgut mit unterschiedlichen Legierungsgehalten verwendet. Diese wurden durch MAG-Schweißen mit Vergütungslagen-Aufbau hergestellt. Abb. 1 zeigt den Querschliff einer solchen Probe im geätzten Zustand. Alle mikrostrukturiellen Untersuchungen wurden in der zuletzt aufgetragenen Schweißlage gemacht, um Effekte durch die Wärmeeinbringung von danach aufgebrachten Schweißlagen ausschließen zu können. Für die Lichtmikroskopie wurden die metallographischen Schliffe mit Diamantsuspension poliert und gemäß Tab. 1 geätzt [10]. Für die EBSD Messungen wurden die Schliffe nach den herkömmlichen Polierschritten zusätzlich zwei Stunden in OPS-Suspension vibropoliert. Die bei den Messungen verwendeten Parameter sind in Tab. 2 zusammengefasst. Bei der Auswertung wurden „inverse pole figure maps" erstellt mit zusätzlicher Anzeige der Bildqualität. Eine ausführliche Erklärung der angewendeten Charakterisierungsmethode EBSD kann in [11] nachgelesen werden.

Es wurde versucht, Ergebnisse aus den Untersuchungen der Mikrostruktur mit der Kerschlagarbeit der Probenzu korrelieren.Für die Kerbschlagbiegeversuche wurden Proben mit V-Kerbe nach Norm DIN EN ISO 15792-1 derart entnommen, dass die Kerbe in der Mitte des Schweißguts liegt. Die Proben wurden gemäß DIN EN ISO 148-1 bei unterschiedlichen Temperaturen geprüft.

\section{Ergebnisse und Diskussion}

\subsection{Lichtmikroskopie}

Nach einer Ätzung mit Pikrinsäure war eine dendritische Struktur sichtbar (Abb. 2). Diese entsteht während der Erstarrung. Aufgrund der Legierungszusammensetzung und der damit erwarteten Erstarrungsbedingungen konnte geschlossen werden, dass es sich hierbei um die primär ent-

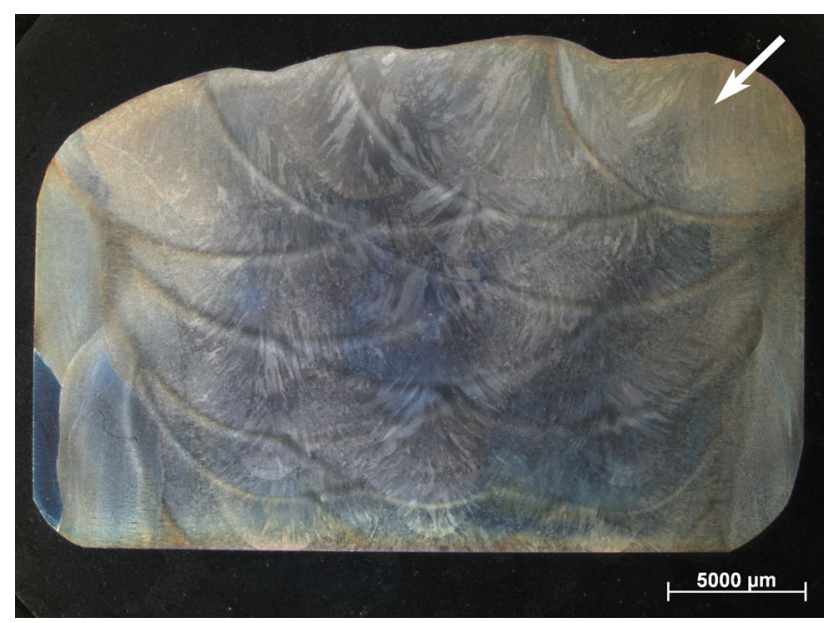

Abb. 1: Querschliff einer Schweißgutprobe im geätzten Zustand. Der Pfeil markiert die zuletzt aufgetragene Schweißlage stehenden $\delta$-Ferrit Dendriten und nicht um die danach entstehende $\gamma$-Austenit Struktur handelt.

Eine Aufnahme derselben Stelle nach einer Nital-Ätzung ist in Abb. 3 dargestellt. Die Nital-Ätzung wird bei bainitischen und martensitischen Stählen üblicherweise zur Sichtbarmachung der Lattenstruktur verwendet. Im Falle des Schweißguts entstand allerdings bei Betrachtung der geätzten Probe mit kleiner Vergrößerung zusätzlich ein deutlicher Kontrast durch Grau-Schattierungen. Eine genauere Untersuchung der unterschiedlich schattierten Bereiche ergab, dass die Schattierung mit der ehemaligen Austenitstruktur in Verbindung gebracht werden kann. So erstreckte sich z. B. eine Martensitlatte niemals über eine Grenze zwischen zwei unterschiedlich schattierten Bereichen. Die längliche Form der Austenitkörner lässt auf eine gerichtete Erstarrung aufgrund eines großen Temperaturgradienten schließen.

Diese zwei Methoden boten sich somit an, um unterschiedlich legierte Proben hinsichtlich der jeweiligen Korngröße auszuwerten und diese danach mit den mechanischen Eigenschaften zu vergleichen. Zuvor wurden jedoch noch EBSD-Messungen durchgeführt, um diese mit den lichtmikroskopischen Versuchen zu korrelieren.

\subsection{EBSD}

Der EBSD Scan von der mit Härteeindrücken markierten Stelle ist in Abb. 4 zu sehen. Jedem gemessenen Punkt wurde gemäß seiner kristallografischen Orientierung nach dem Farbdreieck links unten im Bild eine Farbe zugeordnet („inverse pole figure map“). Die typische Lattenstruktur des Martensits ist deutlich erkennbar. Diese „inverse pole figure map“ wurde über eine lichtmikroskopische Aufnahme nach Nital-Ätzung gelegt. Charakteristische Formen im EBSD-Bild passten exakt zu denen im Nital-Bild. Es kam also zu keinen nennenswerten Verzerrungen mit fortschreitender Dauer des EBSD Scans.

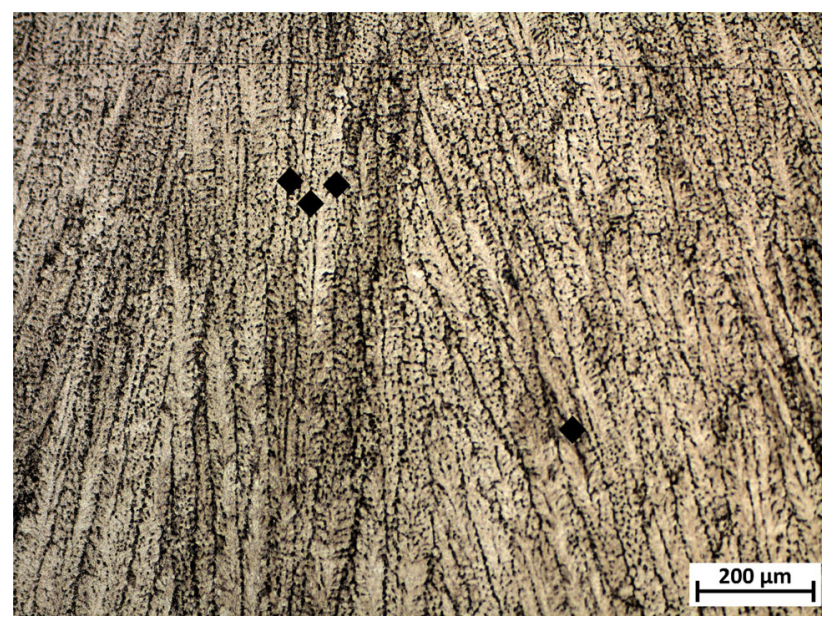

Abb. 2: Dendritische Mikrostruktur nach Ätzung mit Pikrinsäure, aufgenommen im Lichtmikroskop. Die Härteeindrücke dienen zur Markierung der betrachteten Stelle für weiterführende Untersuchungen 


\begin{tabular}{|lll|}
\hline $\begin{array}{l}\text { TABELLE 1: } \\
\text { Chemische Zusammensetzung der verwendeten Ätzmittel [10] }\end{array}$ \\
\hline Ätzmittel & Zusammensetzung & Ätzzeit \\
\hline Pikrinsäure & $100 \mathrm{ml}$ kaltgesättigte Pikrinsäure, $5 \mathrm{ml}$ Agepon, $10 \mathrm{ml}$ Xylol, $0,5 \mathrm{ml} \mathrm{HCl}$ & $60 \mathrm{~s}$ \\
\hline Nital & $3 \% \mathrm{HNO}_{3}$ in Ethanol & $3 \mathrm{~s}$ \\
\hline
\end{tabular}

\begin{tabular}{|ll|}
\hline $\begin{array}{l}\text { TABELLE 2: } \\
\text { Verwendete Parameter für EBSD Messungen }\end{array}$ \\
\hline Spannung & $20 \mathrm{kV}$ \\
\hline Strom & $10 \mathrm{nA}$ \\
\hline Binning & $4 \times 4$ \\
\hline Tilt angle & $70^{\circ}$ \\
\hline Background & Standard \\
\hline Hough Pattern Type & Classic \\
\hline Hough Resolution & Low \\
\hline Convolution Mask & $9 \times 9$ \\
\hline Binned Pattern Size & 120 \\
\hline Theta Step Size & 0,5 \\
\hline
\end{tabular}

Aufgrund der Orientierungs-Beziehung zwischen Austenit und Martensit [4] können innerhalb eines ehemaligen Austenitkorns immer nur ähnliche Farben auftreten. Ein Vergleich der Grau-Schattierungen in Abb. 3 und der Farbbereiche in Abb. 4 zeigte, dass zwar einige ehemalige Austenitkörner durch die Grau-Schattierungen wiedergegeben werden, dass aber in den EBSD Scans zusätzliche ehemalige Austenitkörner sichtbar sind. Eine Nital-Ätzung reicht also nicht aus, das ehemalige Austenitgefüge vollständig zu enthüllen.

Vergleicht man Abb. 4 mit Abb. 2, kann man einen Zusammenhang der Wachstumsrichtung der primären Dendriten und der sekundären Austenitkörner feststellen. Es besteht aber sonst keine Abhängigkeit der Morphologie der Austenitkörner von der Form der primären Dendriten, wes-

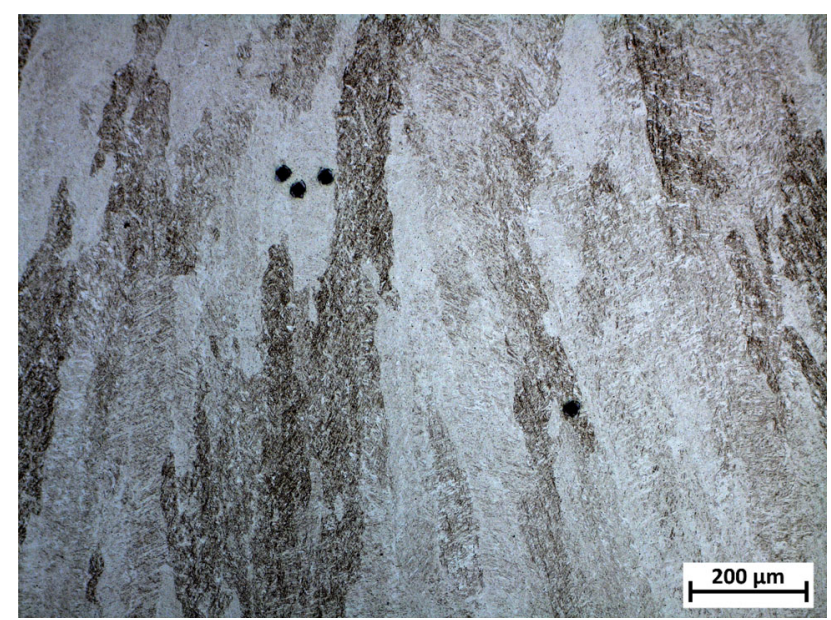

Abb. 3: Lichtmikroskopische Aufnahme der Probe nach einer Nital-Ätzung. Die entstandenen Grau-Schattierungen können mit der ehemaligen Austenitstruktur in Verbindung gebracht werden halb die dendritische Struktur und die Austenitkornstruktur getrennt voneinander zu betrachten sind.

\subsection{Korngrößen und mechanische Eigenschaften}

Abb. 5 zeigt die gemessenen primären und sekundären Dendritenabstände mehrerer Proben mit unterschiedlichem Legierungsgehalt. Zusätzlich ist die Kerbschlagarbeit bei Raumtemperatur und bei $-40^{\circ} \mathrm{C}$ aufgetragen. Der primäre Dendritenabstand ist in allen Fällen größer als der sekundäre Dendritenarmabstand. Es besteht jedoch keine Verbindung zwischen den Dendritenabständen und der Kerbschlagarbeit. Betrachtet man zum Beispiel die Proben S1 und S4, so sind die Dendritenabstände kaum unterschiedlich, die Kerbschlagarbeiten sind aber sehr unterschiedlich. Daraus kann man schließen, dass die Dendritengröße und die damit verbundene interdendritische Seigerung keinen Einfluss auf die Kerbschlagarbeit des Schweißguts haben.

Es muss somit ein anderer Faktor ausschlaggebend sein für die mechanischen Eigenschaften. Es ist altbekannt, dass in martensitischen Stählen die ehemalige Austenitkorngröße maßgeblich für die Kerbschlagarbeit ist. Neuere Untersuchungen zeigten zusätzlich, dass die ehemalige Austenitkorngröße linear von der Größe der "blocks" und „packets" abhängt [7]. Daher ist eine tiefgehende Untersuchung dieser mikrostrukturiellen Einheiten nötig. Es zeigte sich, dass dafür die Lichtmikroskopie mit Nital-Ätzung nicht ausreicht. EBSD lieferte hingegen ein kompletteres Bild der Mikrostruktur. In weiterer Folge soll deshalb mit EBSD

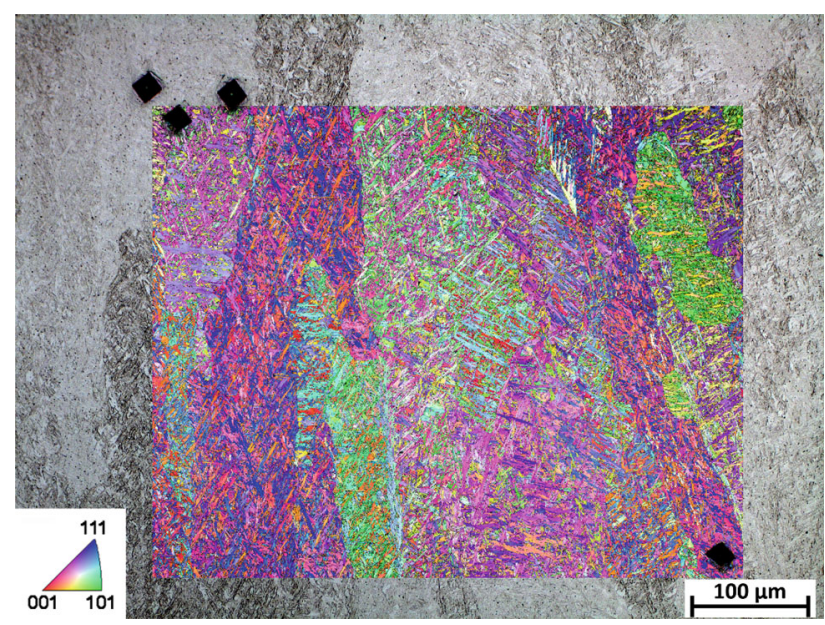

Abb. 4: Bild nach Nital-Ätzung und „inverse pole figure map“ eines EBSD Scans übereinander gelegt 
Abb. 5: Vergleich der primären und sekundären Dendritenabstände mehrerer Proben mit ihrer Kerbschlagarbeit (Charpy-V)

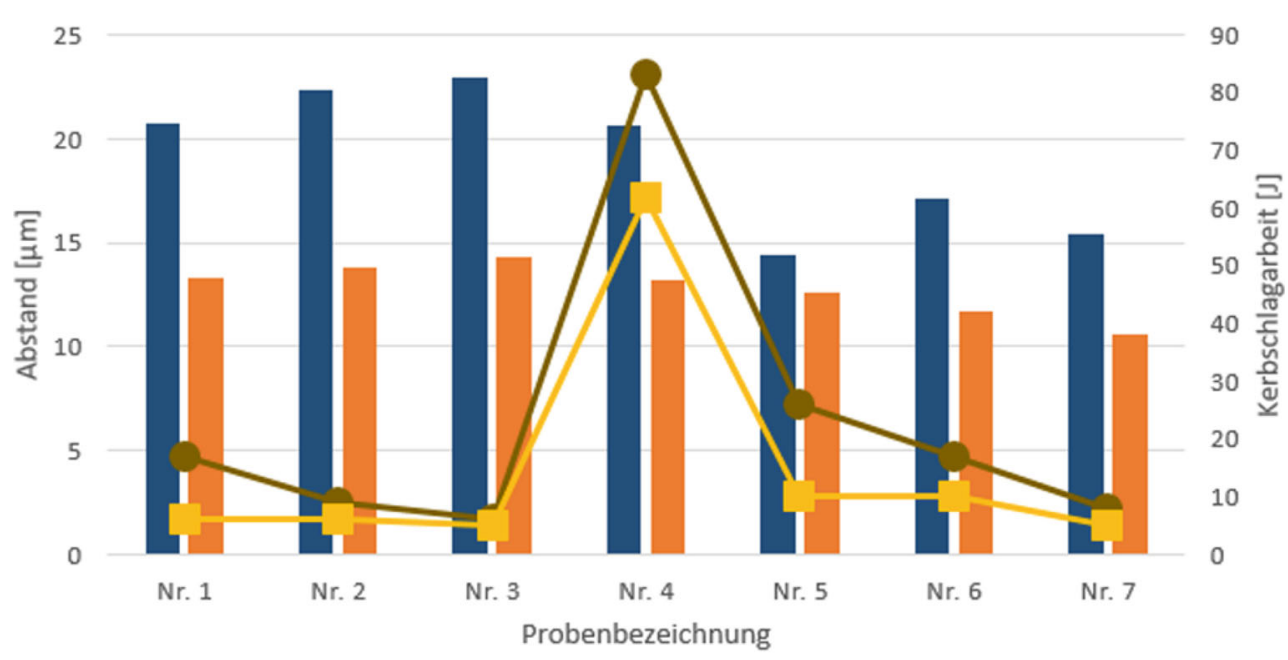

Primärer Dendritenabstand $\quad$ Sekundärer Dendritenarmabstand
Kerbschlagarbeit RT gearbeitet werden, um die daraus gewonnenen Daten mit den mechanischen Eigenschaften korrelieren zu können.

\section{Schlussfolgerungen}

- Die Mikrostruktur von martensitischem Schweißgut wurde mit unterschiedlichen Methoden untersucht.

- Eine Ätzung mit Pikrinsäure bringt die primäre dendritische Struktur von der Erstarrung über $\delta$-Ferrit zum Vorschein. Vergleich man die Dendritengröße mit den mechanischen Eigenschaften, lässt sich kein Zusammenhang erkennen.

- Nach Nital-Ätzung zeigt sich eine Grau-Schattierung, die mit der ehemaligen Austenitstruktur in Verbindung gebracht werden kann.

- EBSD-Untersuchungen zeigten, dass die Grau-Schattierung nach der Nital-Ätzung nicht ausreicht, um die ehemaligen Austenitkörner vollständig zu charakterisieren.

- Weiterführende Versuche mit EBSD sind daher notwendig, um die Mikrostruktur bestmöglich charakterisieren zu können. Danach wird eine neuerliche Korrelation mit den mechanischen Eigenschaften angestrebt.

\section{Danksagung}

Das K-Projekt Network of Excellence for Metal JOINing wird im Rahmen von COMET - Competence Centers for Excellent Technologies durch BMWFW, BMVIT, FFG, Land Oberösterreich, Land Steiermark, Land Tirol und SFG gefördert. Das Programm COMET wird durch die FFG abgewickelt.

Open Access This article is distributed under the terms of the Creative Commons Attribution 4.0 International License (http://creativecommons.org/licenses/by/4.0/), which permits unrestricted use, distribution, and reproduction in any medium, pro- vided you give appropriate credit to the original author(s) and the source, provide a link to the Creative Commons license, and indicate if changes were made.

\section{Literatur}

1. Rauch, R.; Kapl, S.; Posch, G.; Radlmayr, K.: High strength low alloy steel weldments with accommodated qualities to the base metal, BHM, 157 (2012), Nr. 3, S. 102-107

2. Bouyne, E.; Flower, H.M.; Lindley, T.C.; Pineau, A.: Use of EBSD technique to examine microstructure and cracking in a bainitic steel, Scripta Materialia, 39 (1998), Nr. 3, S. 295-300

3. Gourgues, A.F.; Flower, H.M.; Lindley, T.C.: Electron backscatter diffraction study of acicular ferrite, bainite, and martensite steel microstructures, Materials Science and Technology, 16 (2000), S. $26-40$

4. Morito, S.; Tanaka, H.; Konishi, R.; Furuhara, T.; Maki, T.: The morphology and crystallography of lath martensite in Fe-C alloys, Acta Materialia, 51 (2003), S. 1789-1799

5. Morito, S.; Huang, X.; Furuhara, T.; Maki, T.; Hansen, N.: The morphology and crystallography of lath martensite in alloy steels, Acta Materialia, 54 (2006), S. 5323-5331

6. Miyamoto, G.; Takayama, N.; Furuhara, T.: Accurate measurement of the orientation relationship of lath martensite and bainite by electron backscatter diffraction analysis, Scripta Materialia, 60 (2009), S. $1113-1116$

7. Galindo-Nava, E.I.; Rivera-Diaz-del-Castillo, P.E. J.: A model for the microstructure behaviour and strength evolution in lath martensite, Acta Materialia, 98 (2015), S. 81-93

8. Vanovsek, W.; Bernhard, C.; Fiedler, M.; Posch, G.: Influence of aluminum content on the characterization of microstructure and inclusions in high-strength steel welds, Welding in the World, 57 (2013), Nr. 1, S. 73-83

9. Vanovsek, W.; Bernhard, C.; Fiedler, M.; Schnitzer, R.: Effect of titanium content on the solidification and post-solidification microstructure of high-strength steel welds, Welding in the World, 57 (2013), Nr. 5, S. 665-674

10. Petzow, G.: Metallographisches Ätzen, 5. Aufl., Berlin: Gebrüder Borntraeger, 1976

11. Schwartz, A.J.; Kumar, M.; Adams, B. L.; Field, D.P.: Electron backscatter diffraction in materials science, 2. Aufl., New York: Springer, 2009 